\title{
METHOD ON VIRTUAL CROP SYSTEM MODELING BASED ON AGENT TECHNOLOGY
}

\author{
Ping Zheng, Zhongbin Su ${ }^{*}$, Jicheng Zhang, Yujia Zhang \\ Engineering Academy, Northeast Agricultural University, Harbin, Heilongjiang Province, P. \\ R. China 150030 \\ * Corresponding author, Address: Engineering Academy, Northeast Agricultural University, \\ Harbin, Heilongjiang Province, P. R. China 150030. Tel:+86-0451-55190170, Fax:+86- \\ 0451-55190170,Email: suzb001@163.com
}

Abstract: On the basis of adequate understanding the significance of virtual crops research, according to the research state and characteristics of virtual crops' typical modeling methods at home and abroad, a new modeling thoughtway of virtual crops system was put forward based on Agent technology. This method established crops self-learning system based on neural network. It is helpful to understand crop's structural and physiological rule, and to simulate the interaction influnce both between crop and crop and between crop and environment effectively. This research provided a new test platform for Agent technology's verification and perfection which is of great significance academically as well as practically.

Keywords: Agent technology; neural network; virtual plant; interaction influence

\section{INTRODUCTION}

Virtual crops belongs to application basic research, involving agriculture, mathematics, computer graphics, and other related fields. It is of great significance to extract the rules of crops structural and physical information, high-yield plant type design, optimization of field management which is a hot issue in the forefront of agricultural research.

At present, virtual crops research methods include L-system, AMAP, particle systems and three-dimensional reconstruction system, with their own study objects and applictions. All these methods can be summed the

Please use the following format when citing this chapter:

Zheng, P., Su, Z., Zhang, J. and Zhang, Y., 2009, in IFIP International Federation for Information Processing, Volume 294, Computer and Computing Technologies in Agriculture II, Volume 2, eds. D. Li, Z. Chunjiang, (Boston: Springer), pp. 1505-1510. 
law from the morphological characteristics, physical characteristics ,then crop modeling, and dynamic displaying its modeling. But under the existing method of modeling, it can show crop growth process in a specific environment, not the dynamic changes along with environment changes independently. Modeling with these mehods could not simulate the growth of groups crops taking into account less physical features, and plants can not simulate the interactive simulation both between plants, and plants and the environment.

So, according to the research state and characteristics of virtual crops' typical modeling methods at home and abroad, this paper puts forward a new modeling thoughtway of virtual crops system based on Agent technology. This method established crops self-learning system based on neural network. It is helpful to understand crop's structural and physiological rule, and to simulate the interaction influnce both between crop and crop and between crop and environment effectively. This research provided a new test platform for Agent technology's verification and perfection which is of great significance academically as well as practically.

\section{THE ANALYSIS OF BIOLOGICAL SIMULATING RESEARCH BASED ON AGENT}

Resently, agent and multi-agent systems research in the field of artificial intelligence is a very active issue, and has made great progress.

1987, C.W.Reynolds first proposed the animation of bird populations. $\mathrm{He}$ put forward a cluster of computing model, and each bird is an animated role to simulate the dynamic interaction between it and other birds and the environment. Then he put forward restriction simulation on the basis of his group bird simulation before.

MIT multimedia Laboratory designed and developed a virtual dog Silas T. Dog which is a typical representative in the virtual reality filed. Silas's T. Dog lived in an artificial enviornment ALIVE (Artificial Life Interactive Video Environment) system. The system uses hierarchical structure of organizational behavior. Users can directly interact with the virtual dog. The dog's behaviors including acts of self-interest, goaldriven behaviour and so on get more vivid along with the continuous interaction with users

In the study of artificial fish, the most influential one is the Xiaoyuan's Fish. Xiaoyuan's Fish is a computer simulation technology based on biophysical and intelligent behavior model. It also simulates a movable artificial fish social in the virtual ocean. In the society, each Xiaoyuan's Fish has a body driven by variable internal muscle, fin and visual 
perceptible eye. It not only has vivid appearance image like natural fish, but has mental status、 habit perception ability to environment and life characteristic like intention 、reaction 、movement control v purposeful behavior and so on. All those actions are driven by the environmental perception and inner desire, not by key frames and scripts. The fish uses intention generation to produce action programme on the basis of judgement about the environment. Operators need only set the kind and their initial conditions when initialized, and the fish will move independently on their own intention.

Through the study of simulation artificial animals, it is helpful to divide crops growing system and the establish self-study mechanisms for crops model. But there is essential difference between crops and animals, the former is annual plants generally with short growth cycle, widely change range on morphologically and physiologically in one year. Moreover, complex changes in external factors will bring important impact on the crops morphology and physiology. Many factors also control the formation of crop yields, and lead to many key technologic issues exist in virtual crops simulation to be resolved. The study will play virtual crops advantage to support agricultural production.

\section{ESTABLISHMENT VIRTUAL CROPS ARCHITECTURE}

\subsection{Establishment of architecture framework}

Based on the characteristics of agent technology, the paper designs crop growth architecture including perception system 、 cognitive system 、 behavior system, see Fig.1. This architecture can not only reflect to outside environment real-time, make reasonable growing behavior, and feedback to the environment, but can carry out interactive simulation among the morphology, physiology and the environment effectively. Specific features are as follows:

The perception system: it is an information acquisition system, including receptor as to temperature, humidity, light intensity, perception processing module and information syncretize module. In a moment, all kinds of receptors receive and pre-treat the information received, then integrate them in order to prepare for the subsequent information processing and behavior decision.

The cognitive system: it is an information processing system, to process perception information with the output of perception system. It makes behavior decision, then pass its order to the behavior system to execute. 
The behavior system: it is an behavior planning system. It plays a role in the environment directly, including a group of behavior program and a group of effectors. Behavior program is the high-level behavior, the effectors are specific executive actions, and each behavior program is decomposed with a series of low-level action to implement.

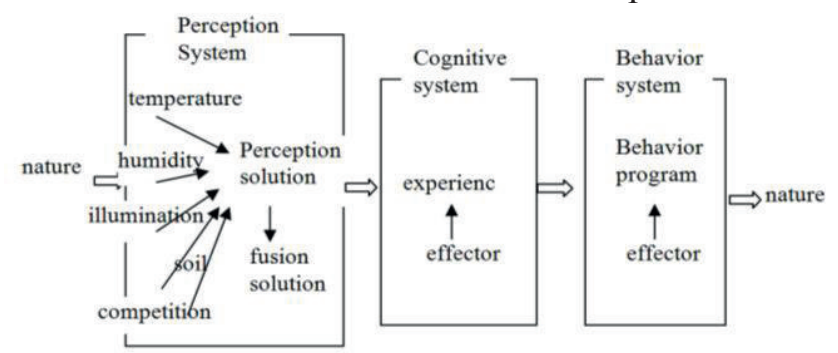

Fig. 1 Virtual corps architecture

\subsection{Virtual crops "self-learning" system based on the neural network}

The plant grows in special environment. Different environmental impact will make crops change their growing status. As a result, crops model not only can real-time montor environmental change to make a reasonable growth; but change normal growth laws according to abnormality in the environment to achieve the purpose of reproduction and evolution. So the crop growth model wanted need to carry out "selflearning" in accordance with the outside environment, respond flexibly and reasonably, accord with the interaction between crops growth and the nature.

Virtual crops "self-learning" system based on the neural network is designed in perception system. The parameters of virtual farmland environment as input lead into the neural network to learn. Learned virtual crops can produce autonomic behavior under guide of cognitive model at the time of changes taken place in the external environment, then effect of action is passed on to neural work. At the same time, fitness increase to some extent will lead to virtual crops better adapt to the changes in the external environment, see Fig.2.

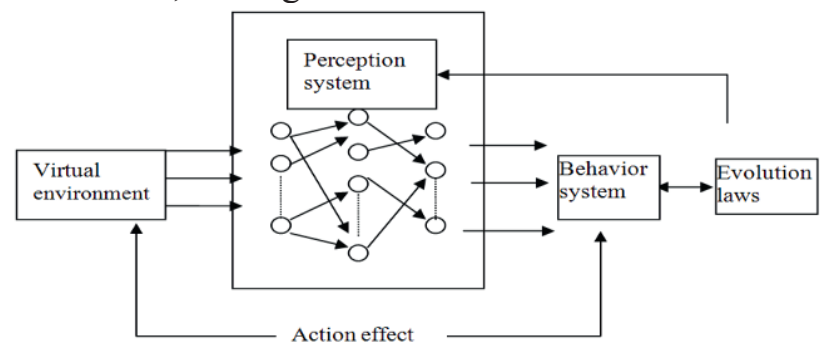

Fig. 2 Virtual crops selp-learning system 


\subsection{Behavior simulation research based on crops population driven by resourse competition}

The paper simulates crops goups by multi-agent involving cooperative law of crop growth on the basis of individual agent establishment and perfection. Usually crops growth belongs to selfish cooperative mode to compete solar energy、water and space among the individuals in order to survive and reproduce better. Agent cooperative behavior belongs to spontaneous cooperation, plan behavior in accordance with their respective objectives, the current state and long-term interests.

Each agent communicates through sending information and receiving coordination among them, which enable each agent can transfer information, ultimately implement cooperation. It is a reason device of communication and coordination mechanism impact on the cooperation efficiency, robustness and expansibility of entire system that it is quite important during devise multi-agent.

Crops make growth planning through the forecast of environmental resources because trigger factor of crops population from environment. In order to maintain the relative performance of agent, index will remain unchanged if the other agent is sharing the same resources. But when the lack of resources affect the survival of the agent, index have to be changed to adapt to the competitive environment. It will be a advantage that we apply agent technology to simulation of virtual crops growth, because the current research methods of virtual crops are all difficult to reach simulation results of groups.

\section{CONCLUSION}

Establish a architecture of virtual crops having learning mechanism, induct and abstract the common features and phenomena of crops growth and development from diversified natural features and phenomena, simulate functions and features of crops self-propagation, adaptive and self-optimizing as a result, manifest crops life characteristics of stability of internal self-stabilization of adapt to the external environment 、 adaptive and self-coordination when agent technology is introduced into model construction of virtual crops. Simulate growth process of competition among crop groups based on distributed artificial intelligence theory, open up a new research method on simulation of virtual crop groups through researching proximity principle、 quality principle and principle of adaptability of crops growth. Architecture of crop individual and groups are established, at the same time, based on simulation advantage of artificial intelligence in simulating crops growth aspects to 
deal with interactive impact between environmental and crops actively and effectively with environmental factors as integral part of simulation of crop growth, which contribute to training and strengthening self-learning system of crop model.

In this paper, we choose virtual crops research based on agent technology on the basis of understand research significance on virtual crops fully and combine with research status and characteristics of virtual crops at home and abroad, provide a new research platform for improving agent research technology because it expands research field of agent technology.

\section{ACKNOWLEDGEMENTS}

This study has been funded by Chinese 863 Plan (\#2006AA10Z231), Program for Innovative Research Team of Northeast Agricultural University, "IRTNEAU"(CXZ010-2) and supported by "211 project".

\section{REFERENCES}

Ban Xiaojuan, Ai Dongmin et al. Artificial fish. Science Publisher. 2007(in Chinese)

Ban Xiaojuan, Liu Hongwei, et al. Study on Self-learning Method of "Artificial Fish". Computer Engineering. 2004.30(6),21-24(in Chinese)

Meng Xianyu, Yin Yixin et al. Research on virtual smelling perception system of artificial fish based on fuzzy reasoning. Journal of System simulation. 2007.19(20),4663-4780(in Chinese)

Su Zhongbin, Meng Fanjiang, Kang Li,et al.Virtual plant modeling based on Agent technology. Agricultural Engineering. 2005, 21 (8): 114- 116. (in Chinese)

$\mathrm{Tu}$ X. Artificial animals for computer animation: biomechanics, locomotion, perception and behavior. University of Toronto, Ph. D thesis, 1996

Zhang Shujun, Ban Xiaojuan, et al. Memory-based Cognitive Model of Artificial Fish. Computer Engineering. 2007.33(19),33-38(in Chinese) 